\title{
Cost-effectiveness of long-acting reversible contraceptive methods: a review
}

\author{
Kusum V. Moray ${ }^{1}$, Himanshu Chaurasia ${ }^{2}$, Beena N. Joshi ${ }^{2 *}$
}

\begin{abstract}
${ }^{1}$ Department of Community Medicine, Ashwini Rural Medical College and Hospital, Kumbhari, Solapur, Maharashtra, India

${ }^{2}$ Health Technology Assessment Resource Hub, Indian Council of Medical Research, National Institute for Research in Reproductive and Child Health, Parel, Mumbai, Maharshtra, India
\end{abstract}

Received: 12 January 2022

Accepted: 02 February 2022

\author{
*Correspondence: \\ Dr. Beena N. Joshi, \\ E-mail: joshib@nirrh.res.in
}

Copyright: ( $)$ the author(s), publisher and licensee Medip Academy. This is an open-access article distributed under the terms of the Creative Commons Attribution Non-Commercial License, which permits unrestricted non-commercial use, distribution, and reproduction in any medium, provided the original work is properly cited.

\begin{abstract}
Long-acting reversible contraception (LARC) methods are proven to be effective in preventing unintended pregnancies. Evidence on cost-effectiveness of these methods will enable policy makers to introduce them in national policies and programs to improve contraceptive access. The aim was to review the studies based on economic evaluations of LARC methods and provide evidence to policy makers to renew their commitment to family planning access. A review of studies on economic evaluations of LARC methods was done. This article uses data from three electronic databases: PubMed, Cochrane and Web of Science to examine whether LARC is cost-effective for clinical trials. The results are presented as a narrative review and summary tables. The literature search yielded 87 studies and 12 studies (five economic evaluations studies from USA) and (five studies from Europe while two studies were from low and middleincome countries) were included. Out of 12 papers, nine had multiple comparators; seven included female sterilization as a comparator, while two studies compared one individual LARC contraceptive method with an individual SARC method. All studies consistently showed that LARCs dominated all SARC (short acting reversible contraceptives) methods. Within LARC, copper IUD, LNG-IUS and implant were more cost-effective than DMPA. After a period of five years, female sterilization turned out to be more cost-effective than LARC methods. LARC methods are costeffective as compared to SARC methods, especially after 1 year of use. Vasectomy is more cost-effective than LARC methods. Policy makers can consider the findings of this review to aid decision making in contraceptive method introduction or scale-up access.
\end{abstract}

Keywords: Long-acting reversible contraceptive, Cost-effectiveness, Short acting reversible contraceptive, Narrative review, Economic evaluation

\section{INTRODUCTION}

There were 1.9 billion women in the reproductive age of 15-49 years in the world in 2019. Of them, 1.1 billion required family planning (FP), 842 million of whom used contraceptive methods and 270 million had unmet need of contraception. ${ }^{1,2}$ Female sterilization and male condoms are preferred the most in the world. $42 \%$ women did not need contraception and $10 \%$ had an unmet need. $27 \%$ used LARC methods which included intra-uterine device
(IUD), copper or levonorgestrel, either combined or progestin-only implants and injectable contraceptive. ${ }^{3}$

Differences in contraceptive prevalence across countries may be attributed to biomedical, socio-cultural and policy aspects. In developed (high-income) countries, women relied mostly on oral contraceptives (OC pills) and condoms. In low and middle-income countries, only 6.5 percent of women used IUD and 1.8 percent used injectable. ${ }^{4}$ In this paper we explored the cost- 
effectiveness of LARC methods to aid governments in making decisions on introducing or scaling up LARC methods.

LARC as contraceptive methods requiring administration less than once per cycle or month. ${ }^{5,6}$ LARC methods like IUDs and subdermal implants and injectables provided a safe and clinically-effective option that could help women space or limit births. ${ }^{7,8}$ Evidence suggested that increased use of LARC methods reduced unintended pregnancy and abortion rates. ${ }^{9}$ While their use in some developed nations was gradually increasing, they remained uncommon in most low- and middle income countries (LMICs). ${ }^{7,10-12}$ Reducing barriers to access of LARC methods may continue to help lower unintended rates of pregnancy. ${ }^{13}$

Lower-middle and low-income countries account for $1 \%$ to $4 \%$ use of implants and IUDs compared to $16 \%$ IUD users in upper middle and $6.5 \%$ in high-income countries. Asia accounts for very low implant users ranging from $0 \%$ in Iran and India to $0.3 \%$ in Pakistan. Most African countries have low prevalence of LARC methods. ${ }^{14}$

Our review on cost-effectiveness studies of LARC was aimed at providing evidence to policy makers of these countries to renew their commitment to FP access. The availability of one contraceptive method to a population enables a four to eight percentage points improvement in contraceptive prevalence. ${ }^{15}$ Cost-effectiveness analysis (CEA) took into account all costs and health effects and helped policy makers to prioritize available alternatives within the limited healthcare resources.

\section{Methods}

A narrative literature review was carried out to find costeffectiveness studies on LARC methods. The review summarized descriptive data specific to 4 LARC devices (etonorgestrel subdermal implant, copper-IUD and levonorgestrel intra-uterine system (LNG-IUS)). The search was conducted using 3 electronic databases: Medline via PubMed, Cochrane and Web of Science for published evidence from January 2000 to August 2020. The list of search terms used is provided in Appendix 1. Only English-language studies were included. PICOT criteria were developed for selection of the studies to be included in the current study. Population of interest was women using LARC method. Comparison of the LARC interventions, to other LARC methods, no method, sterilization, SARC methods or a combination of different methods were included for this review. Only economic evaluation type of studies were included either model based or alongside an RCT or an observational study. Studies that compared individual contraceptive methods or compared different hypothetical scenarios in a model were included. Studies that evaluated the cost-effectiveness of the different timing of insertion of LARC were excluded. Costing studies that did not compare cost-effectiveness of different contraceptives or were not economic evaluation studies were excluded. Reviews on cost-effectiveness of
LARC were excluded for our review; but are referred to in the discussion section of this paper.

Data from included studies were extracted to obtain information on author, year of publication, study settings including country context where economic evaluation was conducted, type of LARC, whether the economic evaluation was done alongside an RCT or COHORT study or whether it was model based and if model based, type and structure of model, model characteristics, measured outcomes and study population characteristics. All studies that were included were evaluated critically using the CHEERS checklist. The checklist had 24 items to assess quality.

\section{Results}

The literature search in the 3 electronic databases yielded 87 studies. After title and abstract screening, 12 papers were selected for the review. A brief summary of the included studies is presented in Table 1.

\section{Study designs of included studies}

Eleven $(91.6 \%)$ of the 12 economic evaluations were model based, with seven of the eleven model type being Markov models. Four of the studies did not specifically mention the type of model used. These studies described the model conceptually, but did not explicitly mention the model type. One of the studies was not model based and the economic evaluation was conducted based on a COHORT study of implanon users. ${ }^{16}$ Six studies considered the societal perspective, four were from health system or provider perspective and two were from thirdparty payer perspective.

\section{Country of origin of authors}

Five $(41.6 \%)$ of the economic evaluations were done by authors based in the USA and five $(41.6 \%)$ of the studies had authors from Europe. Only two (16.6\%) of the studies were by authors from low and middle-income countries, 1 from Iran and another from India.

\section{Comparators}

Nine of the 12 papers had multiple comparators. Five of the economic evaluations compared more than five individual contraceptive methods. Seven of the studies included sterilization as a comparator. Two studies compared 1 individual LARC contraceptive method with an individual SARC method: Implanon as compared to OC pill and LNG-IUS $13.5 \mathrm{mg}$ as compared to OC pill. ${ }^{16,17}$ Only one study compared two scenarios, one with standard of care and the other with etonorgestrel contraceptive implant added to the standard of care. 


\section{Outcomes}

\section{Reported as cost savings}

Five of the 12 studies had defined outcomes as cost savings. The costs of different contraceptive methods were compared and cost savings over the different time horizons were reported.

Chiou et al in 2003 in USA showed that LNG-IUS, copperIUD and 3-monthly injectables were the least expensive with cost-savings of 1646 USD, 1678 USD and 2195 USD per woman per year. ${ }^{18}$

Sonnenbrerg et al showed in 2004 in the USA compared various contraceptive methods to non-use of contraception. ${ }^{19}$ Their results showed that there were cost savings in the range of USD 5907 per woman for tubal sterilization to USD 9936 for vasectomy with a time period of 2 years. All LARC methods had higher cost savings as compared to SARC methods. Copper IUD and LNG-IUS had cost savings of USD 9765 and USD 9763 respectively while oral contraceptive pills had cost saving of USD 8829 ; measured over a 2 year period.

Lipetz et al showed in 2009 in United Kingdom that providing Implanon ${ }^{\circledR}$ was more cost-effective than providing oral contraceptive pills. ${ }^{16}$ Implanon ${ }^{\circledR}$ was half as expensive as oral contraception after using for 1 year.

Foster et al showed in 2013 in USA, that among 11 methods of contraception, intrauterine contraception (both copper and hormonal) and etonorgestrel implants showed the most cost savings. ${ }^{20}$ For every USD spent on users these LARC were able to save 5 USD. For all the 11 methods, the cost of an unintended pregnancy on failure of the method was much higher than the cost of providing the contraception.

Henry et al showed in 2015 in Sweden that use of LNGIUS $13.5 \mathrm{mg}$ resulted in costs savings of 311000 Euros among 1000 women of aged 15-44 years. Also, the study showed that among women using the LNG-IUS, 55 unintended pregnancies were reported as compared to 294 among the women who used oral contraceptive pills.

\section{Reported as cost per couple-years of protection}

Two of the studies reported cost per couple-years of protection (CYP) between comparators.

Philips et al showed in his study, done in Wales in 2000 that the cost per CYP for Implanon ${ }^{\circledR}$ was GBP 95 and the cost per CYP was GBP 168 for LNG-IUS. The injectable contraceptive DMPA showed a cost per CYP of 131; hence, the implant was more cost-effective than the DMPA.

Nakhaee et al study in Iran in 2002. ${ }^{21}$ This study used real world data and one couple year of protection meant that a couple who used the contraceptive method did not conceive for a period of one year. The cost per adjusted couple years of protection was lowest for vasectomy at 10.4 USD and copper IUD at 13.4 USD. It was highest for implants at 82.8 USD.

\section{Reported as incremental cost-effectiveness ratios (ICER)}

Four of the studies had ICER as outcome. One of the studies had incremental cost-utility ratio (ICUR) as outcome.

Varney et al reported from the UK NHS perspective in 2004. ${ }^{22}$ The study showed that using LNG-IUS or etonorgestrel implant was more cost-effective as compared to using DMPA. Also, using the implant instead of LNGIUS was the least cost-effective method. Hence LNG-IUS dominated among the three options.

Mavranezouli et al study in 2008 in UK showed that the ICER of implant versus IUD was GBP 13206 per unintended (UIP) averted over one year of use. ${ }^{23}$ The ICER decreased progressively until at 15 years, the implant became more cost-effective than IUD. The LARC methods were more cost-effective than oral contraception. Female sterilization overtook the LARC methods after five years of use. Among the LARC methods, DMPA and LNG-IUS were not as cost-effective as copper IUD and implants.

Trussel et al study in the USA in 2009 showed that keeping copper-IUD as reference, ICER values of vasectomy, LNG-IUS and implants were 164, 1415, 3828 USD per unintended pregnancy averted. ${ }^{24}$ The rest of the methods including sterilization and SARC were more expensive and less effective than copper-IUD; hence not costeffective.

Trussel et al in 2014 showed that in the USA, LNG-IUS $13.5 \mathrm{mg}$ was more cost-effective as compared to SARC methods comprising of OC pill, ring, patch and contraceptive injections. ${ }^{17}$ Compared to women using SARC methods, who reported higher UIP of 276; women using LNG-IUS $13.5 \mathrm{mg}$ reported 64 UIP. Women using LNG-IUS had lower total costs of $1,283,479$ USD as compared to $1,862,633$ USD among SARC users resulting in a saving of $31 \%$ during the time horizon of three years. This study reports costs and outcomes separately and does not report ICER. We have calculated the ICER from the given information and reported in Table 1.

Joshi et al in 2020 from India used a different approach. ${ }^{25}$ Instead of cost-effectiveness of individual contraceptive methods, cost-effectiveness of adding a new contraceptive method (etonorgestrel implant) to the existing scenario in India was assessed. This study showed that the ICUR of standard of care scenario as compared to addition of Implanon ${ }^{\circledR}$ scenario was 232 USD implying that adding the implant to the public health system of India would be cost-effective. 
Table 1: Included studies in the cost-effectiveness review.

\begin{tabular}{|c|c|c|c|c|c|c|c|c|}
\hline 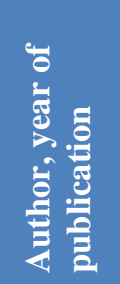 & 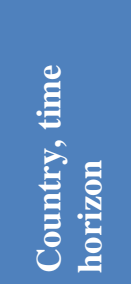 & 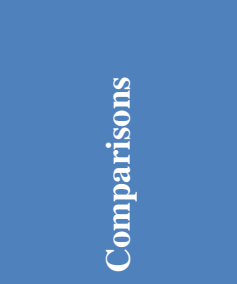 & $\frac{8}{3}$ & 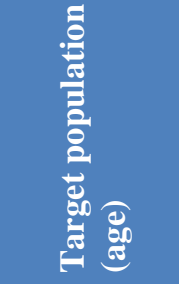 & 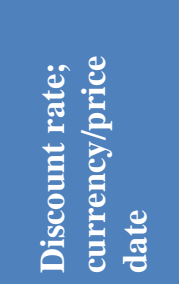 & 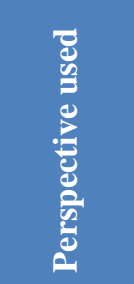 & 茙 & 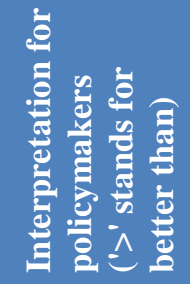 \\
\hline $\begin{array}{l}\text { Philips } \\
2000\end{array}$ & $\begin{array}{l}\text { UK, } \\
\text { Europe }\end{array}$ & $\begin{array}{l}\text { Implanon } \\
\text { versus } \\
\text { Norplant } \\
\text { versus Mirena } \\
\text { versus DMPA }\end{array}$ & $\begin{array}{l}\text { Decision } \\
\text { tree }\end{array}$ & $\begin{array}{l}\text { Not } \\
\text { specified }\end{array}$ & $\begin{array}{l}\text { Discounted } \\
\text { UIP but } \\
\text { not costs }\end{array}$ & $\begin{array}{l}\text { Healthc } \\
\text { are } \\
\text { service }\end{array}$ & $\begin{array}{l}\text { Cost per CYP: } \\
£ 95 \text { for } \\
\text { Implanon; } \\
£ 146 \\
\text { Norplant; } \\
£ 168 \text { for } \\
\text { Mirena and } \\
£ 131 \text { for } \\
\text { DMPA }\end{array}$ & $\begin{array}{l}\text { ImplanonL } \\
\text { NG-IUS } \\
\text { and } \\
\text { ImplanonD } \\
\text { MPA }\end{array}$ \\
\hline $\begin{array}{l}\text { Chiou } \\
\text { et al } \\
2003\end{array}$ & USA & $\begin{array}{l}\text { Nine } \\
\text { contraceptive } \\
\text { methods } \\
\text { (including 3- } \\
\text { month } \\
\text { injectable, oral } \\
\text { contraceptives } \\
\text {, IUD, } \\
\text { intrauterine } \\
\text { system (IUS), } \\
\text { barrier } \\
\text { methods and } \\
\text { surgical } \\
\text { methods) }\end{array}$ & $\begin{array}{l}\text { Markov } \\
\text { model }\end{array}$ & $\begin{array}{l}\text { Women } \\
\text { who do not } \\
\text { intend to } \\
\text { have } \\
\text { children } \\
\text { for the } \\
\text { next five } \\
\text { years }\end{array}$ & $\begin{array}{l}3 \% \text { for } \\
\text { costs }\end{array}$ & $\begin{array}{l}\text { Third- } \\
\text { party } \\
\text { payer }\end{array}$ & $\begin{array}{l}\text { Cost saving } \\
\text { per person for } \\
\text { 5-year: } \$ 1646 \\
\text { for LNG-20 } \\
\text { IUS, } \$ 1678 \\
\text { for Copper-T } \\
380 \text { A and } \\
\$ 2195 \text { for 3- } \\
\text { month } \\
\text { injectable }\end{array}$ & $\begin{array}{l}\text { Copper-T } \\
380 A>\mathrm{LNG} \\
\text {-IUS and } \\
\text { DMPA }>\text { Co } \\
\text { pper-T in } \\
\text { terms of } \\
\text { cost savings }\end{array}$ \\
\hline $\begin{array}{l}\text { Nakha } \\
\text { ee et al } \\
2002\end{array}$ & $\begin{array}{l}\text { Iran; } \\
\text { NR }\end{array}$ & $\begin{array}{l}\text { Seven } \\
\text { Individual } \\
\text { methods } \\
\text { compared: } \\
\text { IUD, } \\
\text { injectable, } \\
\text { OC, condom, } \\
\text { implants, tubal } \\
\text { ligation, } \\
\text { vasectomy }\end{array}$ & $\begin{array}{l}\text { Not } \\
\text { mentione } \\
\text { d }\end{array}$ & $\begin{array}{l}\text { Couples of } \\
\text { reproducti } \\
\text { ve age (not } \\
\text { specified). }\end{array}$ & $\begin{array}{l}\text { Not } \\
\text { applied; } \\
\text { US\$2000 }\end{array}$ & $\begin{array}{l}\text { Provide } \\
\mathrm{r} \\
\text { perspec } \\
\text { tive } \\
\text { (institut } \\
\text { ional) }\end{array}$ & $\begin{array}{l}\text { Cost per CYP: } \\
\text { vasectomy } \\
\text { cheapest } \\
\$ 10.4, \text { IUD } \\
\$ 13.4, \text { OCP } \\
\$ 21.1, \\
\text { condoms } \\
\text { \$24.1, tubal } \\
\text { ligation \$27.8, } \\
\text { injectables } \\
\text { \$46.8, } \\
\text { implants } \$ 82.8\end{array}$ & $\begin{array}{l}\text { Vasectomy } \\
>\text { Copper- } \\
\text { IUD and } \\
\text { Implanon } \\
\text { has least } \\
\text { cost saving }\end{array}$ \\
\hline $\begin{array}{l}\text { Sonne } \\
\text { nberg } \\
\text { et al } \\
2004\end{array}$ & $\begin{array}{l}\text { USA; } \\
\text { two } \\
\text { years }\end{array}$ & $\begin{array}{l}13 \text { methods } \\
\text { compared to } \\
\text { non-use: COC, } \\
\text { transdermal } \\
\text { contraceptive } \\
\text { patch, vaginal } \\
\text { ring, male } \\
\text { condom, } \\
\text { diaphragm, } \\
\text { copper IUD, } \\
\text { LNG-IUS, } \\
\text { DMPA, } \\
\text { oestrogen- } \\
\text { progestin } \\
\text { monthly }\end{array}$ & $\begin{array}{l}\text { Markov } \\
\text { model }\end{array}$ & $15-50$ & $\begin{array}{l}3 \% ; 2002 \\
\text { USD }\end{array}$ & $\begin{array}{l}\text { Societa } \\
1 \\
\text { perspec } \\
\text { tive }\end{array}$ & $\begin{array}{l}\text { Cost savings } \\
\text { for } 2 \text { years } \\
\text { versus no } \\
\text { method: } 9765 \\
\text { USD for } \\
\text { copper IUD; } \\
9763 \text { USD for } \\
\text { LNG-IUS; } \\
\text { 9815 USD for } \\
\text { DMPA }\end{array}$ & $\begin{array}{l}\text { Similar cost } \\
\text { savings for } \\
\text { copper-IUD } \\
\text { and LNG- } \\
\text { IUS }\end{array}$ \\
\hline
\end{tabular}




\begin{tabular}{|c|c|c|c|c|c|c|c|c|}
\hline 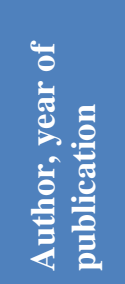 & 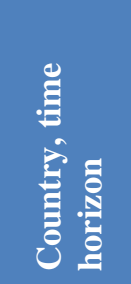 & 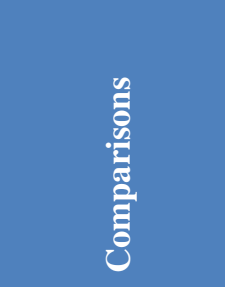 & $\frac{8}{\frac{8}{2}}$ & 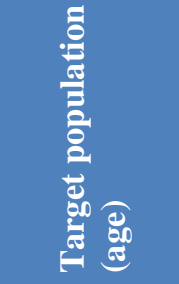 & 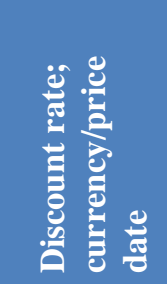 & 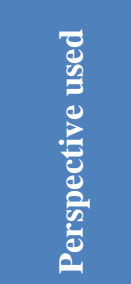 & 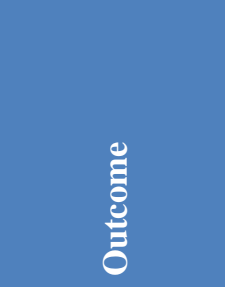 & 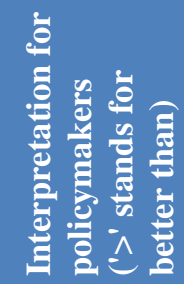 \\
\hline & & $\begin{array}{l}\text { injectable, } \\
\text { periodic } \\
\text { abstinence, } \\
\text { withdrawal, } \\
\text { tubal } \\
\text { sterilisation, } \\
\text { vasectomy }\end{array}$ & & & & & & \\
\hline $\begin{array}{l}\text { Varne } \\
\text { y et al } \\
2004\end{array}$ & $\begin{array}{l}\text { UK; } \\
\text { UK } \\
\text { NHS; } \\
\text { varied }\end{array}$ & $\begin{array}{l}\text { Depo-Provera, } \\
\text { Implanon, } \\
\text { Mirena }\end{array}$ & $\begin{array}{l}\text { Not } \\
\text { mentione } \\
d\end{array}$ & $>30$ & $\begin{array}{l}3.5 \% ; \\
2002 / 2003 \\
\text { GBP }\end{array}$ & $\begin{array}{l}\text { Societa } \\
1 \\
\text { perspec } \\
\text { tive }\end{array}$ & $\begin{array}{l}\text { ICER of } £ 20 \\
953 \text { per } \\
\text { additional } \\
\text { pregnancy } \\
\text { averted for } \\
\text { Mirena versus } \\
\text { Implanon (in } \\
\text { favour of } \\
\text { Mirena) }\end{array}$ & $\begin{array}{l}\text { LNG-IUS > } \\
\text { Implanon }\end{array}$ \\
\hline $\begin{array}{l}\text { Mavra } \\
\text { nezouli } \\
2008\end{array}$ & $\begin{array}{l}\text { UK; } 15 \\
\text { years }\end{array}$ & $\begin{array}{l}\text { LARC versus } \\
\text { COC versus } \\
\text { female } \\
\text { sterilization }\end{array}$ & $\begin{array}{l}\text { Markov } \\
\text { model }\end{array}$ & $\begin{array}{l}\text { Reproducti } \\
\text { ve age (not } \\
\text { specified) }\end{array}$ & $\begin{array}{l}3.5 \% \\
2005 \text { GBP }\end{array}$ & $\begin{array}{l}\text { Societa } \\
1 \\
\text { perspec } \\
\text { tive } \\
\text { (UK } \\
\text { NHS) }\end{array}$ & $\begin{array}{l}\text { ICER of } \\
\text { implant versus } \\
\text { IUD was } £ 13 \\
206 \text { per } \\
\text { pregnancy } \\
\text { averted at one } \\
\text { year of use; } \\
\text { implant } \\
\text { dominates } \\
\text { IUD at } 15 \\
\text { years. } \\
\text { ICER of £38 } \\
\text { 197 per } \\
\text { pregnancy } \\
\text { averted for } \\
\text { Oral } \\
\text { contraceptive } \\
\text { versus LARC } \\
\text { (favouring } \\
\text { LARC). } \\
\text { After six years } \\
\text { Female } \\
\text { sterilization } \\
\text { dominated all } \\
\text { LARC }\end{array}$ & $\begin{array}{l}\text { Implanon > } \\
\text { IUD at } 15 \\
\text { years and } \\
\text { LARC> } \\
\text { oral } \\
\text { contraceptio } \\
\mathrm{n} \text { and } \\
\text { Female } \\
\text { sterilization } \\
>\text { LARC at } \\
6 \text { years of } \\
\text { use }\end{array}$ \\
\hline $\begin{array}{l}\text { Lipetz } \\
\text { et al } \\
2009\end{array}$ & $\begin{array}{l}\text { Wales } \\
\text { commu } \\
\text { nity; } \\
\text { three } \\
\text { years }\end{array}$ & $\begin{array}{l}\text { Implanon } \\
\text { versus OC pill }\end{array}$ & $\begin{array}{l}\text { Alongsid } \\
\text { e a } \\
\text { cohort } \\
\text { study; } \\
\text { not } \\
\text { model- } \\
\text { based }\end{array}$ & $\begin{array}{l}\text { Not } \\
\text { specified }\end{array}$ & $\begin{array}{l}\text { None; } \\
\text { none } \\
(\mathrm{GBP})\end{array}$ & $\begin{array}{l}\text { Annual } \\
\text { health } \\
\text { costs } \\
\text { (Wales } \\
\text { NHS) }\end{array}$ & $\begin{array}{l}\text { Cost per } \\
\text { patient per } \\
\text { cumulative } \\
\text { years of use at } \\
\text { completion of } \\
\text { three years of } \\
\text { use: } £ 50 \text { in } \\
\text { Implanon } \\
\text { versus } £ 83 \text { for } \\
\text { Oral } \\
\text { contraception }\end{array}$ & $\begin{array}{l}\text { Implanon }>0 \\
\text { ral } \\
\text { contraceptio } \\
\mathrm{n} \text { in terms } \\
\text { of cost } \\
\text { savings }\end{array}$ \\
\hline
\end{tabular}




\begin{tabular}{|c|c|c|c|c|c|c|c|c|}
\hline 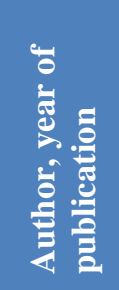 & 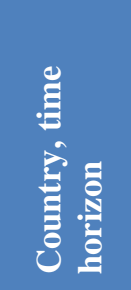 & 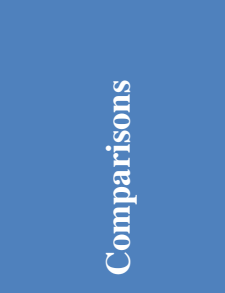 & $\frac{8}{\frac{8}{0}}$ & 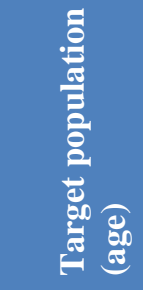 & 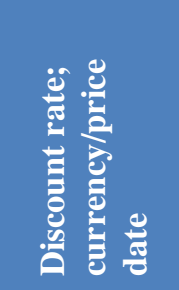 & 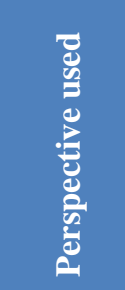 & $\begin{array}{l}\stackrel{\mathscr{E}}{8} \\
\stackrel{8}{3} \\
\end{array}$ & 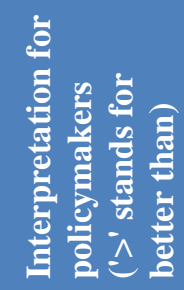 \\
\hline $\begin{array}{l}\text { Trusse } \\
\text { ll et al } \\
2009\end{array}$ & $\begin{array}{l}\text { USA; } \\
\text { health } \\
\text { care } \\
\text { payer; } \\
\text { five } \\
\text { years }\end{array}$ & $\begin{array}{l}16 \text { individual } \\
\text { methods } \\
\text { compared with } \\
\text { non use: } \\
\text { IUDs, } \\
\text { sterilisation, } \\
\text { condoms, } \\
\text { implants, } \\
\text { behavioural, } \\
\text { other barrier, } \\
\text { OC, } \\
\text { transdermal } \\
\text { patch, } \\
\text { injectable, no } \\
\text { contraception }\end{array}$ & $\begin{array}{l}\text { Markov } \\
\text { model }\end{array}$ & $\begin{array}{l}\text { Not } \\
\text { specified }\end{array}$ & $\begin{array}{l}3 \% \text { to } \\
\text { costs; } 2007 \\
\text { USD }\end{array}$ & $\begin{array}{l}\text { Societa } \\
1 \\
\text { perspec } \\
\text { tive }\end{array}$ & $\begin{array}{l}\text { All ICERs } \\
\text { relative to } \\
\text { copper IUD: } \\
\text { vasectomy } \\
\$ 164 \text { per one } \\
\text { point } \\
\text { reduction in } \\
\text { the annual } \\
\text { probability of } \\
\text { pregnancy; } \\
\text { LNG-IUS } \\
\$ 1415 ; \\
\text { implants } \\
\text { \$3828; all } \\
\text { other methods } \\
\text { dominated by } \\
\text { copper IUD }\end{array}$ & $\begin{array}{l}\text { vasectomy> } \\
\text { LNG-IUS } \\
\text { and Implant } \\
\text { with } \\
\text { reference to } \\
\text { copper IUD }\end{array}$ \\
\hline $\begin{array}{l}\text { Foster } \\
\text { et al } \\
2013\end{array}$ & $\begin{array}{l}\text { USA; } \\
\text { two } \\
\text { years }\end{array}$ & $\begin{array}{l}\text { Individual } \\
\text { methods } \\
\text { compared: } \\
\text { Interval tubal } \\
\text { ligation, tubal } \\
\text { occlusion, } \\
\text { copper IUC, } \\
\text { hormonal } \\
\text { IUC, } \\
\text { Implanon, } \\
\text { injectable, } \\
\text { ring, patch, } \\
\text { OC, barriers, } \\
\text { emergency } \\
\text { contraceptives }\end{array}$ & $\begin{array}{l}\text { Not } \\
\text { mentione } \\
\text { d }\end{array}$ & $15-44$ & $\begin{array}{l}\text { None; } \\
2009 \text { USD }\end{array}$ & $\begin{array}{l}\text { Public } \\
\text { health } \\
\text { progra } \\
\text { m (not } \\
\text { clear) }\end{array}$ & $\begin{array}{l}\text { Cost savings } \\
\text { per dollar } \\
\text { expenditure: } \\
\text { Highest for } \\
\text { Implant, } \\
\text { Copper IUD } \\
\text { and Hormonal } \\
\text { IUD at } 5,5 \\
\text { and } 4.89 \text { USD } \\
\text { respectively }\end{array}$ & $\begin{array}{l}\text { Implant=Co } \\
\text { pper-IUD> } \\
\text { Hormonal } \\
\text { IUD in } \\
\text { terms of } \\
\text { cost savings }\end{array}$ \\
\hline $\begin{array}{l}\text { Trusse } \\
\text { ll et al } \\
2014\end{array}$ & $\begin{array}{l}\text { USA; } \\
\text { instituti } \\
\text { onal; } \\
\text { three } \\
\text { years }\end{array}$ & $\begin{array}{l}\text { LNG-IUS } \\
13.5 \mathrm{mg} \text { vs } \\
\text { SARCs }\end{array}$ & $\begin{array}{l}\text { Markov } \\
\text { model }\end{array}$ & $22-29$ & $\begin{array}{l}3 \% \text { to } \\
\text { costs; } 2012 \\
\text { USD }\end{array}$ & $\begin{array}{l}\text { Third- } \\
\text { party } \\
\text { payer }\end{array}$ & $\begin{array}{l}\text { ICER of } 2731 \\
\text { USD per } \\
\text { Unintended } \\
\text { pregancy } \\
\text { averted in } \\
\text { favour of } \\
\text { LNG-13.5mg- } \\
\text { IUS as } \\
\text { compared to } \\
\text { SARC }\end{array}$ & $\begin{array}{l}\text { LNG- } \\
13.5 \mathrm{mg} \\
\text { IUS> } \\
\text { SARC }\end{array}$ \\
\hline $\begin{array}{l}\text { Henry } \\
\text { et al } \\
2015\end{array}$ & $\begin{array}{l}\text { Sweden } \\
\text {; three } \\
\text { years }\end{array}$ & $\begin{array}{l}\text { Novel LNG- } \\
\text { IUS ( } 13.5 \mathrm{mg}) \\
\text { versus oral } \\
\text { contraceptive }\end{array}$ & $\begin{array}{l}\text { Markov } \\
\text { model }\end{array}$ & $15-44$ & $\begin{array}{l}3 \% ; \\
\text { converted } \\
\text { to Euros } \\
27 / 1 / 15\end{array}$ & $\begin{array}{l}\text { Societa } \\
1 \\
\text { perspec } \\
\text { tive } \\
\text { includi } \\
\text { ng } \\
\text { direct } \\
\text { and } \\
\text { indirect } \\
\text { costs }\end{array}$ & $\begin{array}{l}\text { ICER of } \\
€ 1302.7 \text { per } \\
\text { Unintended } \\
\text { pregnancy } \\
\text { averted and } € \\
230629 \text { per } \\
\text { QALY gained; } \\
\text { both in favour } \\
\text { of LNG-IUS } \\
\text { as compared }\end{array}$ & $\begin{array}{l}\text { LNG- } \\
13.5 \mathrm{mg}> \\
\text { oral } \\
\text { contraceptio } \\
\mathrm{n}\end{array}$ \\
\hline
\end{tabular}




\begin{tabular}{|llllllll}
\hline \\
\end{tabular}

Table 2: Critical appraisal of included studies.

\begin{tabular}{|c|c|c|c|c|c|c|c|c|c|c|c|c|c|}
\hline 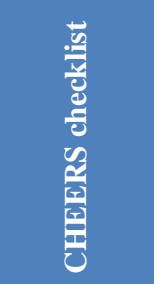 & 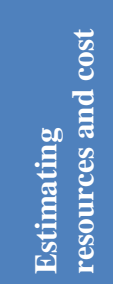 & 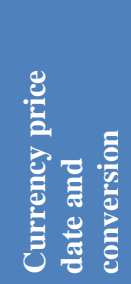 & 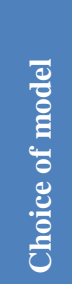 & 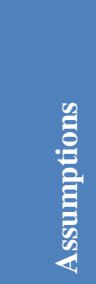 & 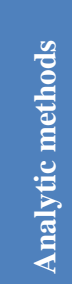 & 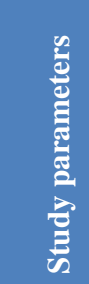 & 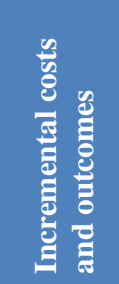 & 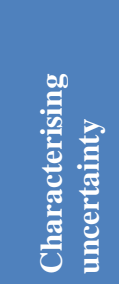 & 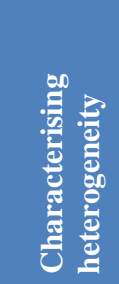 & 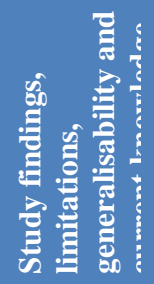 & 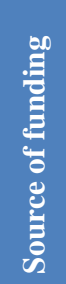 & 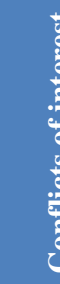 & 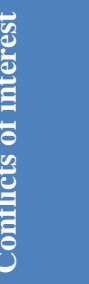 \\
\hline $\begin{array}{l}\text { Philips } \\
2000\end{array}$ & $\mathrm{Y}$ & $\mathrm{N}$ & $\mathrm{N}$ & $\mathrm{Y}$ & $\mathrm{Y}$ & $\mathrm{Y}$ & $\mathrm{N}$ & SW & SW & $\mathrm{Y}$ & $\mathrm{N}$ & $\mathrm{N}$ & Poor \\
\hline $\begin{array}{l}\text { Nakhaee } \\
\text { et al } 2002\end{array}$ & $\mathrm{Y}$ & $\mathrm{Y}$ & $\mathrm{Y}$ & $\mathrm{Y}$ & $\mathrm{Y}$ & $\mathrm{Y}$ & $\mathrm{N}$ & SW & SW & SW & $\mathrm{Y}$ & $\mathrm{N}$ & Poor \\
\hline $\begin{array}{l}\text { Sonnenbe } \\
\text { rg et al } \\
2004\end{array}$ & $\mathrm{~N}$ & $\mathrm{Y}$ & $\mathrm{Y}$ & $\mathrm{Y}$ & $\mathrm{Y}$ & $\mathrm{Y}$ & $\mathrm{N}$ & SW & SW & $\mathrm{Y}$ & $\mathrm{Y}$ & $\mathrm{N}$ & Poor \\
\hline $\begin{array}{l}\text { Varney et } \\
\text { al } 2004\end{array}$ & $\mathrm{Y}$ & $\mathrm{Y}$ & $\mathrm{Y}$ & $\mathrm{Y}$ & $\mathrm{Y}$ & $\mathrm{Y}$ & $\mathrm{Y}$ & $\mathrm{Y}$ & $\mathrm{Y}$ & $\mathrm{Y}$ & $\mathrm{N}$ & $\mathrm{N}$ & Fair \\
\hline $\begin{array}{l}\text { Mavrane } \\
\text { zouli } \\
2008\end{array}$ & $\mathrm{Y}$ & $\mathrm{Y}$ & $\mathrm{Y}$ & $\mathrm{Y}$ & $\mathrm{Y}$ & $\mathrm{Y}$ & $\mathrm{Y}$ & $\mathrm{Y}$ & $\mathrm{Y}$ & $\mathrm{Y}$ & $\mathrm{Y}$ & $\mathrm{N}$ & Good \\
\hline $\begin{array}{l}\text { Lipetz et } \\
\text { al } 2009\end{array}$ & $\mathrm{Y}$ & $\mathrm{N}$ & $\mathrm{N}$ & $\mathrm{Y}$ & $\mathrm{Y}$ & $\mathrm{Y}$ & $\mathrm{N}$ & $\mathrm{Y}$ & $\mathrm{Y}$ & $\mathrm{N}$ & $\mathrm{N}$ & $\mathrm{N}$ & Poor \\
\hline $\begin{array}{l}\text { Trussell } \\
\text { et al } 2009\end{array}$ & $\mathrm{Y}$ & $\mathrm{Y}$ & $\mathrm{Y}$ & $\mathrm{Y}$ & $\mathrm{Y}$ & $\mathrm{Y}$ & $\mathrm{Y}$ & $\mathrm{Y}$ & $\mathrm{Y}$ & $\mathrm{Y}$ & $\mathrm{Y}$ & $\mathrm{N}$ & Good \\
\hline $\begin{array}{l}\text { Foster et } \\
\text { al } 2013\end{array}$ & $\mathrm{Y}$ & $\mathrm{Y}$ & $\mathrm{N}$ & $\mathrm{Y}$ & $\mathrm{Y}$ & $\mathrm{Y}$ & $\mathrm{N}$ & $\mathrm{Y}$ & $\mathrm{Y}$ & $\mathrm{Y}$ & $\mathrm{N}$ & $\mathrm{N}$ & Poor \\
\hline $\begin{array}{l}\text { Trussell } \\
\text { et al } 2014\end{array}$ & $\mathrm{Y}$ & $\mathrm{Y}$ & $\mathrm{Y}$ & $\mathrm{Y}$ & $\mathrm{Y}$ & $\mathrm{Y}$ & $\mathrm{Y}$ & $\mathrm{Y}$ & $\mathrm{Y}$ & $\mathrm{Y}$ & $\mathrm{Y}$ & $\mathrm{Y}$ & Good \\
\hline $\begin{array}{l}\text { Henry et } \\
\text { al } 2015\end{array}$ & $\mathrm{~N}$ & $\mathrm{Y}$ & $\mathrm{Y}$ & $\mathrm{Y}$ & $\mathrm{Y}$ & $\mathrm{Y}$ & $\mathrm{N}$ & $\mathrm{Y}$ & $\mathrm{Y}$ & $\mathrm{N}$ & $\mathrm{Y}$ & $\mathrm{Y}$ & Fair \\
\hline $\begin{array}{l}\text { Joshi et } \\
\text { al } 2020\end{array}$ & $\mathrm{Y}$ & $\mathrm{Y}$ & $\mathrm{Y}$ & $\mathrm{Y}$ & $\mathrm{Y}$ & $\mathrm{Y}$ & $\mathrm{Y}$ & $\mathrm{Y}$ & $\mathrm{Y}$ & $\mathrm{Y}$ & $\mathrm{Y}$ & $\mathrm{Y}$ & Good \\
\hline $\begin{array}{l}\text { Chiou et } \\
\text { al } 2003\end{array}$ & $\mathrm{Y}$ & $\mathrm{Y}$ & $\mathrm{Y}$ & $\mathrm{Y}$ & $\mathrm{Y}$ & $\mathrm{Y}$ & $\mathrm{Y}$ & $\mathrm{Y}$ & $\mathrm{Y}$ & $\mathrm{Y}$ & $\mathrm{Y}$ & $\mathrm{N}$ & Fair \\
\hline
\end{tabular}

\section{Summarizing results on cost-effectiveness of LARC}

The 6 studies that reported outcomes in terms of cost savings showed the following: LARC cost-savings were higher than SARC; however, cost saving in vasectomy was highest. Out of 11 contraceptive methods, most cost savings occurred in copper-IUD and implants. LNG-IUS $13.5 \mathrm{mg}$ was more cost-effective as compared to oral contraceptive pills, contraceptive ring, patch and injectable contraception. There were more cost-savings in the use of LNG-IUS as compared to oral contraceptives. The two studies that reported results as cost per couple years of 
protection showed that implants were more cost-effective than LNG-IUS and DMPA. Cost per CYP was lowest for vasectomy and copper-IUD and highest for implant. The studies that reported ICERs showed the following: LNGIUS or implant was more cost-effective than DMPA. LNG-IUS was more cost-effective than implant. LARC was more cost-effective than SARC; but female sterilization turned out to be more cost-effective than LARC after five years of use. Among LARC, copper-IUD and implant were more cost-effective than LNG-IUS.

Hence from all the above it was consistently observed that LARC was more cost-effective than SARC. Sterilization became more cost-effective than LARC after five years of use. But there was some inconsistency as to which LARC method was most cost-effective. Copper-IUD emerged as cost-effective, however between LNG-IUS and implants studies reported differently as to which was more costeffective. Of the 12 studies, 8 studies considered the etonorgestrel contraceptive implant (Implanon $\AA$ ); three studies did not mention the type of implant and one considers Norplant (levonorgestrel implant).

\section{Critical appraisal of included studies}

All included studies were evaluated critically and the appraisal was presented in Table 2. Studies have been marked as good, fair and poor quality based on their scores. Out of 24, a score of less than 18 was considered poor; 19-22 was fair and 23-24 as good. Five (41.7\%) of the included studies were of poor quality, four $(33.3 \%)$ were of good quality and three $(25 \%)$ were of fair quality.

\section{DISCUSSION}

This review aimed at summarizing cost-effectiveness information on LARC including copper-IUD, LNG-IUS, implants and DMPA. Literature search revealed that there were only a handful of studies on this subject. Most of the included studies have demonstrated cost savings by using contraception as compared to no-contraception and have further shown that LARC methods generate higher cost savings. Only two studies report cost per couple years of protection. One of these studies showed highest cost per CYP for implant. This was the only study that showed implant to be not cost-effective. This was probably because the study used real-word data from Iran in 2002 and the least number of users were for the implant $(n=257)$ while other methods had much higher number of acceptors such as condoms (2077540) and OC pill (197487). Only four studies reported ICERs. One study reported costs and outcome differences separately but did not compute ICER. Only one study from India assessed cost-effectiveness of adding implant to existing scenario and found that addition of implant to the public health system of India was costeffective. Of the 12 studies included in the review, majority (10 of 12 or $83.3 \%$ ) were by authors from developed countries.
In 2018, Lynch et al reviewed the cost-effectiveness of LARC in the Australian context in a narrative review. ${ }^{26}$ They chose studies that reported cost per pregnancy averted. They aimed to review literature to assess if it was generalizable to the Australian context. They also assessed the quality of the 20 included studies using the CHEERS checklist. Overall the review concluded that LARC methods were more cost-effective than oral contraception but highlighted that there were limitations in study quality. Nine studies were common between our review and the review done in 2018. The additional 11 studies included in their review were either older than the year 2000 or did not fit our PICOTs criteria. Of the 20 studies that Lynch et al reviewed; the average score on the CHEERS checklist was $62 / 100$, showing that the overall quality of studies was low. The reasons for this include non-availability of data on aspects of contraception like failure and discontinuation rates. Nine of the 20 included studies in their review derived most of their data from two reference studies using data from the American national survey of family growth. These two studies reported data that was one to two decades old. The review concluded that although many of the included studies were not of the best methodological quality, it was likely that, LARCs were cost-effective from a policy perspective and that LARCs were more costeffective as compared to oral contraceptives.

Despite consistency across studies that LARC were costeffective; their acceptance rates were very variable. In countries like India LARC use accounted to $<5 \%$ and female sterilization accounted to $75 \%$ of contraceptive method mix. A few Central Asian and African countries like Egypt report IUDs to comprise more than $50 \%$ of the method mix. Implants were yet to be used in the public health system of countries like India, while a few African countries like Burkina Faso, Benin, Senegal, Mali and Ghana had $>25 \%$ of their contraceptive users using implants. $^{27}$ The contraceptive method mix in the USA showed that roughly $25 \%$ each were OCP users and sterilization. However there has been a steady increase in LARC users from $6 \%$ in 2008 to $14 \%$ in $2014 .{ }^{28}$ Overall contraceptive use of the world showed that IUDs and implants account to less than 20\%; DMPA accounted to $8 \%$, pills to $16 \%$. Female sterilization and male condoms were the commonest methods used in the world $24 \%$ and $21 \%$ respectively. ${ }^{29}$

It was evident that the difference in availability and prevalence of contraceptive use depended on policies adopted and implemented by different countries. The health systems in different countries varied greatly as well; ranging from completely publicly-funded and regulated health systems to health systems that relied heavily on private and un-regulated markets. It was hence important from a policy perspective that the most cost-effective options among contraceptives like copper-IUD, LNG-IUS and implants be available to all women who needed it in all countries of the world. The contraceptive prevalence report by United Nations for 2019, showed that prevalence of IUD use (the report combines copper IUD and LNG- 
IUS into one category) was less than $1 \%$ in 43 African countries and three Asian countries. Prevalence of implants (the report considers all types of contraceptive implants including combination and progestin-only implants) was less than $1 \%$ in 20 African countries and 36 Asian countries. This showed that there was a large scope for improving access and uptake of contraceptives.

This review can be used as an evidence compilation of cost-effectiveness of LARC methods where the IUD, IUS and the implant consistently turn out cost-effective. This could be used to influence policy in several countries. However, the outcomes reported in these included studies were different (cost-savings, cost per CYP and ICER) and hence statistically combining these outputs was not feasible. Also, most studies were from high-income countries, with only 2 studies from low and middle income countries. The included studies reported their findings in different currencies (pounds, US dollar). We have not converted or inflated these values to current year. The two reasons for this were: the studies were very heterogeneous to statistically combine the findings; intra-study comparison of LARC was meeting the objective of our review not necessitating inter-study comparison. Perspectives used in the models and the type of health systems in the context of the studies were heterogonous as well. Hence countries could either conduct their own economic evaluation of LARC or use this review's findings to bring about positive changes in their contraceptive policy. $41.7 \%$ of included studies were of poor quality as per the CHEERs checklist. This needed to be considered while decision-making.

In countries where all the cost-effective LARC were available but there was low acceptance of LARC; reasons needed to explored. The reasons may vary from lack of access, to lack of information or choice of women to avoid side-effects of hormonal methods. It was hence important to understand the needs and preferences of women locally and ensure that the unmet need of contraceptives was brought down to zero.

A few current international programs that supported commitments to focus and address the unmet need for contraception were the sustainable development goals (SDG), every woman every child (EWEC) and family planning 2020 (FP2020) agenda 2030. The progress that had been achieved in increasing access to family planning needed to be monitored to identify gaps in health systems, funding and research. Of the 17 SDGs and various targets, 1 of them was the target 3.7 that stated ensure universal access to sexual and reproductive health (SRH) services, including FP, by 2030. EWEC aimed at ending preventable deaths of women, children and adolescents and ensuring their safety and well-being; this required universal access to SRH services and rights by the year 2030. FP2020 seeks to extend access of FP to at least 120 million women and girls in some of the world's poorest countries by the year 2020, aiming for a significant increase from the baseline year of $2012 . .^{30-32}$ In 2019,49 percent of women in the reproductive age range (15-49 years) (a total of 22 million women) used some form of contraception worldwide, an increase from 42 percent in 1990 (a total of 554 million women). In 2019, contraceptive use among reproductive-age women was over 55 percent in 37 countries and in 23 countries it was below 20 percent. ${ }^{33}$ These commitments have enabled an increase in contraceptive prevalence over the past few decades and yet there was a huge unmet need for contraception, especially spacing methods. ${ }^{34}$ These continued efforts with back up of evidence on costeffectiveness will help expanding the basket of contraceptive choices and achieve SDGs translating to better quality of lives for women and their families.

\section{CONCLUSION}

All studies consistently showed that LARCs dominated all SARC methods. Within LARC, copper IUD, LNG-IUS and implant were more cost-effective as compared to DMPA. Vasectomy was found to be more cost-effective as compared to LARC and female sterilization was more cost-effective than LARC after five years' time duration.

\section{Recommendations}

The recommendations were to utilize evidence to include the three cost-effective LARC methods: copper-IUD, LNG-IUS and implants in the health system. Acceptability and feasibility studies were prerequisites to program introduction strategies that could help achieve improved uptake of LARC methods.

\section{ACKNOWLEDGEMENTS}

We sincerely thank Ex-Director NIRRCH, Dr. Smita Mahale for encouraging us to take up HTA subject for research and providing necessary support and encouragement.

We thank support extended by HTAIn DHR in allocating topics for conduct of HTA.

Funding: Funded by the department of health research, Ministry of Health and Family Welfare, Government of India

Conflict of interest: None declared

Ethical approval: Not required

\section{REFERENCES}

1. United Nations. Fact sheet: Department of economic and social affairs, population division. Family planning and the 2030 agenda for sustainable development. New York: United Nations. 2019. Available https://www.un.org/en/development/desa/population/ publications/pdf/family/familyPlanning_DataBooklet _2019.pdf. Accessed on 28 December 2021. 
2. Kantorová V, Wheldon MC, Ueffing P, Dasgupta AN. Estimating progress towards meeting women's contraceptive needs in 185 countries: A Bayesian hierarchical modelling study. PLoS Med. 2020;17(2):1003026.

3. WHO. Fact sheet: Family Planning-a global handbook for providers. Available at: https://www.who.int/reproductivehealth/publications /fp-global-handbook/en/. Accessed on 28 December 2021.

4. United Nations. Fact sheet: Department of economic and social affairs. population division. World contraceptive by method, 2019. Available at: https://www.un.org/development/desa/pd/sites/www. un.org.development.desa.pd/files/files/documents/20 20/Jan/un_2019_contraceptiveusebymethod_databoo klet.pdf. Accessed on 28 December 2021.

5. Mavranezouli I. The cost-effectiveness of long-acting reversible contraceptive methods in the UK: analysis based on a decision-analytic model developed for a National institute for health and clinical excellence (NICE) clinical practice guideline. Human Reproduct. 2008;23(6):1338-45.

6. Kolawole OO, Sowemimo OO, Ojo OO, Fasubaa OB. Contraceptive implants: a review and current perspective in southwest Nigeria. Tropic J Obstetr Gynaecol. 2018;35(2):108-12.

7. Staveteig S, Maliick L, Winter R. Uptake and discontinuation of long acting reversible contraceptives in low-income countries. USAID ICF Int. 2015:1-59.

8. Curtis KM, Peipert JF. Long-acting reversible contraception. N E J Med. 2017;376(5):461-8.

9. Blumenthal PD, Voedisch A, Gemzell-Danielsson K. Strategies to prevent unintended pregnancy: increasing use of long-acting reversible contraception. Human Reproduct Update. 2011;17(1):121-37.

10. Adetunji JA. Rising popularity of injectable contraceptives in sub-Saharan Africa. Afr Populat Stud. 2011;25(2).

11. Darroch JE, Singh S. Trends in contraceptive need and use in developing countries in 2003, 2008 and 2012: an analysis of national surveys. Lancet. 2013;381(9879):1756-62.

12. Tibaijuka L, Odongo R, Welikhe E, Mukisa W, Kugonza L, Busingye I, et al. Factors influencing use of long-acting versus short-acting contraceptive methods among reproductive-age women in a resource-limited setting. BMC Women Health. 2017; 17(1):25.

13. Trussell J, Hassan F, Lowin J, Law A, Filonenko A. Achieving cost-neutrality with long-acting reversible contraceptive methods. Contraception. 2015;91(1):49-56.

14. United Nations. Fact sheet: Contraceptive use by method 2019: data booklet, 2019 Available at: https://www.un-ilibrary.org/population-anddemography/contraceptive-use-by-method2019_1bd58a10-en. Accessed on 28 December 2021.
15. Ross J, Stover J. Use of modern contraception increases when more methods become available: analysis of evidence from 1982-2009. Glob Health Sci Pract. 2013;1(2):203-12.

16. Lipetz C, Phillips CJ, Fleming CF. The costeffectiveness of a long-acting reversible contraceptive (Implanon®) relative to oral contraception in a community setting. Contraception. 2009;79(4):304-9.

17. Trussell J, Hassan F, Henry N, Pocoski J, Law A, Filonenko A. Cost-effectiveness analysis of levonorgestrel-releasing intrauterine system (LNGIUS) $13.5 \mathrm{mg}$ in contraception. Contraception. 2014;89(5):451-9.

18. Chiou CF, Trussell J, Reyes E, Knight K, Wallace J, Udani J, et al. Economic analysis of contraceptives for women. Contraception. 2003;68(1):3-10.

19. Sonnenberg FA, Burkman RT, Hagerty CG, Speroff L, Speroff T. Costs and net health effects of contraceptive methods. Contraception. 2004;69(6):447-59.

20. Foster DG, Biggs MA, Malvin J, Bradsberry M, Darney P, Brindis CD. Cost-savings from the provision of specific contraceptive methods in 2009. Women Health Issue. 2013;23(4):265-71.

21. Islamic Republic of Iran. Assessing the costeffectiveness of contraceptive methods in Shiraz. Available

at: https://apps.who.int/iris/handle/10665/119137?locale -attribute $=$ es\&locale $=$ fr. Accessed on 28 December 2021.

22. Relative cost effectiveness of Depo-provera, Implanon and Mirena in reversible long-term hormonal contraception in the UK. Available at: https://www.crd.york.ac.uk/CRDWeb/ShowRecord.a sp?ID=22005008019\&ID=22005008019. Accessed on 28 December 2021.

23. Mavranezouli I, LARC Guideline Development Group. The cost-effectiveness of long-acting reversible contraceptive methods in the UK: analysis based on a decision-analytic model developed for a National Institute for Health and Clinical Excellence (NICE) clinical practice guideline. Hum Reprod. 2008;23(6):1338-45.

24. Trussell J, Lalla AM, Doan QV, Reyes E, Pinto L, Gricar J. Cost effectiveness of contraceptives in the United States. Contraception. 2009;79(1):5-14.

25. Joshi B, Moray KV, Sachin O, Chaurasia H, Begum $\mathrm{S}$. Cost effectiveness of introducing etonorgestrel contraceptive implant into india's current family welfare programme. Appl Health Econ Health Policy. 2020.

26. Lynch M, Lourenco RDA, Flattery M, Haas M. Reviewing the cost-effectiveness of long-acting reversible contraceptive methods in an Australian context. Austral N Z J Obstetr Gynaecol. 2019;59(1):21-35.

27. Family Planning 2020. Data Dashboard, 2020. Available http://www.familyplanning2020.org/data-dashboard. Accessed on 29 December 2021. 
28. Kavanaugh ML, Jerman J. Contraceptive method use in the United States: trends and characteristics between 2008, 2012 and 2014. Contraception. 2018;97(1):14-21.

29. United Nations. Fact sheet: Contraceptive use by method 2019: data booklet, 2019. Available at: https://www.un-ilibrary.org/population-anddemography/contraceptive-use-by-method2019_1bd58a10-en. Accessed on 29 December 2021.

30. Brown W, Druce N, Bunting J, Radloff S, Koroma D, Gupta S, et al. Developing the " 120 by 20 " goal for the Global FP2020 Initiative. Stud Fam Plan. 2014;45(1):73-84.

31. Every Woman Every Child. The global strategy for women's, children's and adolescents health (20162030), 2015. Available at: http://globalstrategy.everywomaneverychild.org/. Accessed on 29 December 2021.
32. United Nations. General Assembly. Transforming our world: the 2030 agenda for sustainable development. resolution adopted by the general assembly on 25 September 2015 (A/RES/70/1). New York (NY): United Nations, 2015. Available at: https://sustainabledevelopment.un.org/post2015/trans formingourworld. Accessed on 29 December 2021.

33. United Nations. Fact sheet: World Fertility and Family Planning, 2020. Highlights (ST/ESA/SER.A/440). 2020.

34. Juarez F, Gayet C, Mejia-Pailles G. Factors associated with unmet need for contraception in Mexico: evidence from the National Survey of Demographic Dynamics 2014. BMC Pub Health. 2018;18.

Cite this article as: Moray $\mathrm{KV}$, Chaurasia $\mathrm{H}$, Joshi BN. Cost-effectiveness of long-acting reversible contraceptive methods: a review. Int J Reprod Contracept Obstet Gynecol 2022;11:997-1008. 
APPENDIX 1: CHEERS checklist evaluation of quality of included studies.

\begin{tabular}{|c|c|c|c|c|c|c|c|c|c|c|c|c|}
\hline $\begin{array}{l}\text { CHEERS } \\
\text { checklist }\end{array}$ & $\begin{array}{l}\text { Title- } \\
1\end{array}$ & $\begin{array}{l}\text { Abstract- } \\
2\end{array}$ & $\begin{array}{l}\text { Backgrou } \\
\text { nd and } \\
\text { objectives }\end{array}$ & $\begin{array}{l}\text { Target } \\
\text { populatio } \\
\text { n and } \\
\text { sub- } \\
\text { groups }\end{array}$ & $\begin{array}{l}\text { Setting } \\
\text { and } \\
\text { locatio } \\
\text { n }\end{array}$ & $\begin{array}{l}\text { Study } \\
\text { perspecti } \\
\text { ve }\end{array}$ & $\begin{array}{l}\text { Comparato } \\
\text { rs }\end{array}$ & $\begin{array}{l}\text { Time } \\
\text { horizo } \\
\text { n }\end{array}$ & $\begin{array}{l}\text { Discou } \\
\text { nt rate }\end{array}$ & $\begin{array}{l}\text { Choice } \\
\text { of } \\
\text { outcom } \\
\text { es }\end{array}$ & $\begin{array}{l}\text { Measureme } \\
\text { nt of } \\
\text { effectivenes } \\
\mathrm{s}\end{array}$ & $\begin{array}{l}\text { Measureme } \\
\text { nt and } \\
\text { valuation of } \\
\text { preference- } \\
\text { based } \\
\text { outcomes }\end{array}$ \\
\hline Philips 2000 & $\mathrm{Y}$ & $\mathrm{N}$ & $\mathrm{Y}$ & $\mathrm{N}$ & $\mathrm{Y}$ & $\mathrm{N}$ & $\mathrm{Y}$ & SW & $\mathrm{N}$ & $\mathrm{Y}$ & $\mathrm{N}$ & $\mathrm{Y}$ \\
\hline $\begin{array}{l}\text { Nakhaee et al } \\
2002\end{array}$ & $\mathrm{Y}$ & $\mathrm{Y}$ & $\mathrm{Y}$ & $\mathrm{N}$ & $\mathrm{Y}$ & $\mathrm{Y}$ & $\mathrm{Y}$ & $\mathrm{N}$ & $\mathrm{N}$ & $\mathrm{Y}$ & $\mathrm{N}$ & $\mathrm{Y}$ \\
\hline $\begin{array}{l}\text { Sonnenberg et al } \\
2004\end{array}$ & $\mathrm{~N}$ & $\mathrm{~N}$ & $\mathrm{~N}$ & $\mathrm{Y}$ & $\mathrm{Y}$ & $\mathrm{N}$ & $\mathrm{Y}$ & $\mathrm{Y}$ & $\mathrm{Y}$ & $\mathrm{Y}$ & $\mathrm{N}$ & $\mathrm{Y}$ \\
\hline $\begin{array}{l}\text { Varney et al } \\
2004\end{array}$ & $\mathrm{Y}$ & $\mathrm{Y}$ & $\mathrm{Y}$ & $\mathrm{Y}$ & $\mathrm{Y}$ & $\mathrm{Y}$ & $\mathrm{Y}$ & $\mathrm{N}$ & $\mathrm{Y}$ & $\mathrm{Y}$ & SW & $\mathrm{Y}$ \\
\hline $\begin{array}{l}\text { Mavranezouli } \\
2008\end{array}$ & $\mathrm{Y}$ & $\mathrm{Y}$ & $\mathrm{Y}$ & $\mathrm{N}$ & $\mathrm{Y}$ & $\mathrm{Y}$ & $\mathrm{Y}$ & $\mathrm{Y}$ & $\mathrm{Y}$ & $\mathrm{Y}$ & $\mathrm{Y}$ & $\mathrm{Y}$ \\
\hline Lipetz et al 2009 & $\mathrm{Y}$ & $\mathrm{Y}$ & $\mathrm{Y}$ & $\mathrm{N}$ & $\mathrm{Y}$ & $\mathrm{Y}$ & $\mathrm{Y}$ & $\mathrm{Y}$ & $\mathrm{N}$ & SW & $\mathrm{N}$ & $\mathrm{Y}$ \\
\hline $\begin{array}{l}\text { Trussell et al } \\
2009\end{array}$ & $\mathrm{Y}$ & $\mathrm{Y}$ & $\mathrm{Y}$ & $\mathrm{N}$ & $\mathrm{Y}$ & $\mathrm{Y}$ & $\mathrm{Y}$ & $\mathrm{Y}$ & $\mathrm{Y}$ & $\mathrm{Y}$ & $\mathrm{Y}$ & $\mathrm{Y}$ \\
\hline Foster et al 2013 & $\mathrm{~N}$ & $\mathrm{~N}$ & $\mathrm{~N}$ & $\mathrm{Y}$ & $\mathrm{N}$ & $\mathrm{N}$ & $\mathrm{Y}$ & $\mathrm{Y}$ & $\mathrm{N}$ & $\mathrm{Y}$ & $\mathrm{N}$ & $\mathrm{Y}$ \\
\hline $\begin{array}{l}\text { Trussell et al } \\
2014\end{array}$ & $\mathrm{Y}$ & $\mathrm{Y}$ & $\mathrm{Y}$ & $\mathrm{Y}$ & $\mathrm{Y}$ & $\mathrm{Y}$ & $\mathrm{Y}$ & $\mathrm{Y}$ & $\mathrm{Y}$ & $\mathrm{Y}$ & $\mathrm{Y}$ & $\mathrm{Y}$ \\
\hline Henry et al 2015 & $\mathrm{Y}$ & $\mathrm{Y}$ & $\mathrm{Y}$ & $\mathrm{Y}$ & $\mathrm{Y}$ & $\mathrm{Y}$ & SW & $\mathrm{Y}$ & $\mathrm{Y}$ & $\mathrm{Y}$ & $\mathrm{N}$ & $\mathrm{Y}$ \\
\hline Joshi et al 2020 & $\mathrm{Y}$ & $\mathrm{Y}$ & $\mathrm{Y}$ & $\mathrm{Y}$ & $\mathrm{Y}$ & $\mathrm{Y}$ & $\mathrm{Y}$ & $\mathrm{Y}$ & $\mathrm{Y}$ & $\mathrm{Y}$ & $\mathrm{Y}$ & $\mathrm{Y}$ \\
\hline Chiou et al 2003 & SW & $\mathrm{Y}$ & $\mathrm{Y}$ & $\mathrm{N}$ & $\mathrm{N}$ & $\mathrm{Y}$ & $\mathrm{N}$ & $\mathrm{Y}$ & $\mathrm{Y}$ & $\mathrm{Y}$ & SW & $\mathrm{Y}$ \\
\hline
\end{tabular}

